\title{
Sums of finite products of Pell polynomials in terms of hypergeometric functions
}

\author{
Asim Patra* ${ }^{*}$ and Gopal Krishna Panda
}

\section{${ }^{*}$ Correspondence:}

asimp1993@gmail.com;

515ma3016@nitrkl.ac.in

Department of Mathematics,

National Institute

of Technology, Rourkela,

Odisha 769008, India

\begin{abstract}
In this work, we study sums of finite products of Pell polynomials and express them in terms of some special orthogonal polynomials. Furthermore, each of the obtained expression is represented as linear combinations of classical polynomials involving hypergeometric functions by means of explicit computations.
\end{abstract}

Keywords: Pell polynomials, Chebyshev polynomials, Hypergeometric functions, Finite products

\section{Introduction}

In the recent years, the problem of expressing sums of products of certain special polynomials in terms of other special polynomials has drawn the attention of many researchers and mathematicians due to which this field has seen an increasing interest [1-3]. There are many special polynomials out of which we deal with the Pell polynomials [46]. The Pell polynomials $P_{k}(t)$ are defined by the binary recurrence relation

$$
P_{k}(t)=2 t P_{k-1}(t)+P_{k-2}(t), P_{0}(t)=0, P_{1}(t)=1, k \geq 2 .
$$

In this work, the following summations of finite products of Pell polynomials have been considered, given by

$$
\sum_{j_{1}+j_{2}+\cdots+j_{r+1}=n} P_{j_{1}+1}(x) P_{j_{2}+1}(x) \ldots P_{j_{r+1}+1}(x)
$$

where the summation runs over all nonnegative integers $j_{1}, j_{2}, \ldots, j_{r+1}$, with $j_{1}+j_{2}+\cdots+j_{r+1}=n$. The summation (2) is represented in terms of some orthogonal polynomials such as the Legendre polynomials $\left(\mathcal{P}_{n}(x)\right)$, Jacobi polynomials $\left(\mathcal{P}_{n}^{\alpha, \beta}(x)\right)$, Hermite polynomials $\left(H_{n}(x)\right)$, Gegenbauer polynomials $\left(C_{n}^{(\lambda)}(x)\right)$, extended Laguerre polynomials $\left(L_{n}^{\alpha}(x)\right)$, and Chebyshev polynomials [7] of first kind $\left(T_{n}(x)\right)$, second kind $\left(U_{n}(x)\right)$, third kind $\left(V_{n}(x)\right)$ and fourth kind $\left(W_{n}(x)\right)$ which are further represented as hypergeometric functions. The hypergeometric function, denoted by ${ }_{2} F_{1}(a, b ; c ; z)$, is basically a special function represented by a hypergeometric series, which involves many special functions as specific cases which has been discussed in the preliminaries section.

The motivation of this work has been derived by the classical linearization problem,

The Author(s) 2022. Open Access This article is licensed under a Creative Commons Attribution 4.0 International License, which permits use, sharing, adaptation, distribution and reproduction in any medium or format, as long as you give appropriate credit to the original author(s) and the source, provide a link to the Creative Commons licence, and indicate if changes were made. The images or other third party material in this article are included in the article's Creative Commons licence, unless indicated otherwise in a credit line to the material. If material is not included in the article's Creative Commons licence and your intended use is not permitted by statutory regulation or exceeds the permitted use, you will need to obtain permission directly from the copyright holder. To view a copy of this licence, visit http:// creativecommons.org/licenses/by/4.0/. 


$$
s_{a}(t) p_{b}(t)=\sum_{k=0}^{a+b} d_{a b}(k) q_{k}(t),
$$

which involves the determination of the unknown coefficients $d_{a b}(k)$ in the expansion of product of the polynomials $s_{a}(t)$ and $p_{b}(t)$ in terms of arbitrary polynomial $\left\{q_{k}(t)\right\}_{k \geq 0}$. If the two polynomials $s_{a}(t)$ and $p_{a}(t)$ are equal to $q_{a}(t)$, then problem (3) is known as the standard linearization problem or Clebsch-Gordan-type. In other words, for $s_{a}(t)=p_{a}(t)=q_{a}(t),(3)$ converts to

$$
s_{a}(t) s_{b}(t)=\sum_{k=0}^{a+b} d_{a b}(k) s_{k}(t)
$$

which is called the Clebsch-Gordan-type problem. Furthermore, if we take $p_{b}(t)=1$, then (3) is called the connection problem expressed as

$$
s_{a}(t)=\sum_{k=0}^{a} d_{a}(k) q_{k}(t)
$$

In addition, if $s_{a}(t)=t^{n}$ in (3), then it is known as the inversion problem.

In particular, the present work is motivated by the linearization problem and may be viewed as a generalization of the classical linearization problem as in (3). Apart from that, our work is also motivated by the convolution identity of Bernoulli polynomials $\mathcal{B}_{n}(x)$ that yields the famous Miki's identity and Faber-Pandharipande-Zagier identity. In other words, it is possible to represent the sums of products of two Bernoulli polynomials as linear combinations of Bernoulli polynomials. The polynomials $\mathcal{B}_{n}(x)$ are given by

$$
\sum_{k=0}^{\infty} \mathcal{B}_{k}(x) \frac{t^{n}}{n !}=\frac{t e^{t x}}{e^{t}-1}
$$

Now, if for $k \geq 2$,

$$
\delta_{k}(x)=\sum_{l=1}^{k-1} \frac{\mathcal{B}_{l}(x) \mathcal{B}_{k-l}(x)}{l(k-l)},
$$

then, from [8],

$$
\begin{gathered}
\sum_{l=1}^{k-1} \frac{\mathcal{B}_{2 l}(x) \mathcal{B}_{2 k-2 l}(x)}{2 l(2 k-2 l)}+\frac{2 \mathcal{B}_{1}(x) \mathcal{B}_{2 k-1}(x)}{(2 k-1)}=\frac{1}{k} H_{2 k-1} \mathcal{B}_{2 l}(x) \\
+\frac{1}{k} \sum_{l=1}^{k}\left(\begin{array}{c}
2 k \\
2 l
\end{array}\right) \frac{1}{2 l} \mathcal{B}_{2 l}(x) \mathcal{B}_{2 k-2 l}(x)+\frac{2 \mathcal{B}_{2 k-1} \mathcal{B}_{1}(x)}{(2 k-1)}
\end{gathered}
$$

can be derived from the Fourier expansion of $\delta_{k}(\langle x\rangle)$, where $\langle x\rangle$ is the fractional part of any real number $x$ expressed as $\langle x\rangle=x-[x]$ and $H_{k}$ are the harmonic numbers denoted by $H_{k}=\sum_{n=1}^{k} \frac{1}{n}$. Furthermore, it is interesting to observe that for $x=\frac{1}{2}$, (8) gives the Faber-Pandharipande-Zagier identity [9] and for $x=0$, (8) gives a slight variant of Miki's identity [10-12]. In this article, the Pell polynomials are represented in terms of 
linear combination of some of the orthogonal polynomials. The generating function of the Pell polynomials is given by

$$
\sum_{n=0}^{\infty} P_{n}(x) t^{n}=\frac{1}{1-2 x t-t^{2}}
$$

First few terms of the Pell polynomials derived from (1) can be written as $P_{2}(t)=2 t, \quad P_{3}(t)=4 t^{2}+1, \quad P_{4}(t)=8 t^{3}+4 t, \quad P_{5}(t)=16 t^{4}+12 t^{2}+1$, $P_{6}(t)=32 t^{5}+32 t^{3}+6 t, \ldots$. The first few terms of $P_{n}(t)$ are graphically depicted in Fig. 1 .

Furthermore, the Pell polynomials are the natural extension of the Pell numbers $P_{n}$ defined by the same recurrence relation $[6,13]$

$$
P_{k}=2 P_{k-1}+P_{k-2}, P_{0}=0, P_{1}=1, k \geq 2 .
$$

The methodology used in this work is beneficial over other techniques in the literature due to the simple Fourier series expansions used here to determine the unknown coefficients involved in the classical linearization type problem unlike the other methods in $[10,11]$ which are quite complex in their approach. The literature survey includes the work of Zhang [7], where he derived a new identity for the Chebyshev polynomials. In [14], the authors have shown an application of a p-adic convolution using a suitable polynomial. In a different work, Kim et al. [2] have studied regarding the sums of finite products of Chebyshev polynomials and Fibonacci polynomials. Apart from that, the sums of finite products of the Genocchi functions have also been studied by Kim et al. [8]. In two different works, Kim et al. tackled with the sums of finite products of Chebyshev and Lucas-balancing polynomials $[15,16]$. Furthermore, certain identities relating to the symmetry for the Euler polynomials are derived in [17]. In [18], a difference of sums of finite products have been tackled in case of Lucas-balancing polynomials. Moreover, the Appell polynomials were utilized to represent a family of associated sequences [19]. Apart from that, a new class of Bernoulli polynomials have been introduced by [20], related to polyexponential functions.

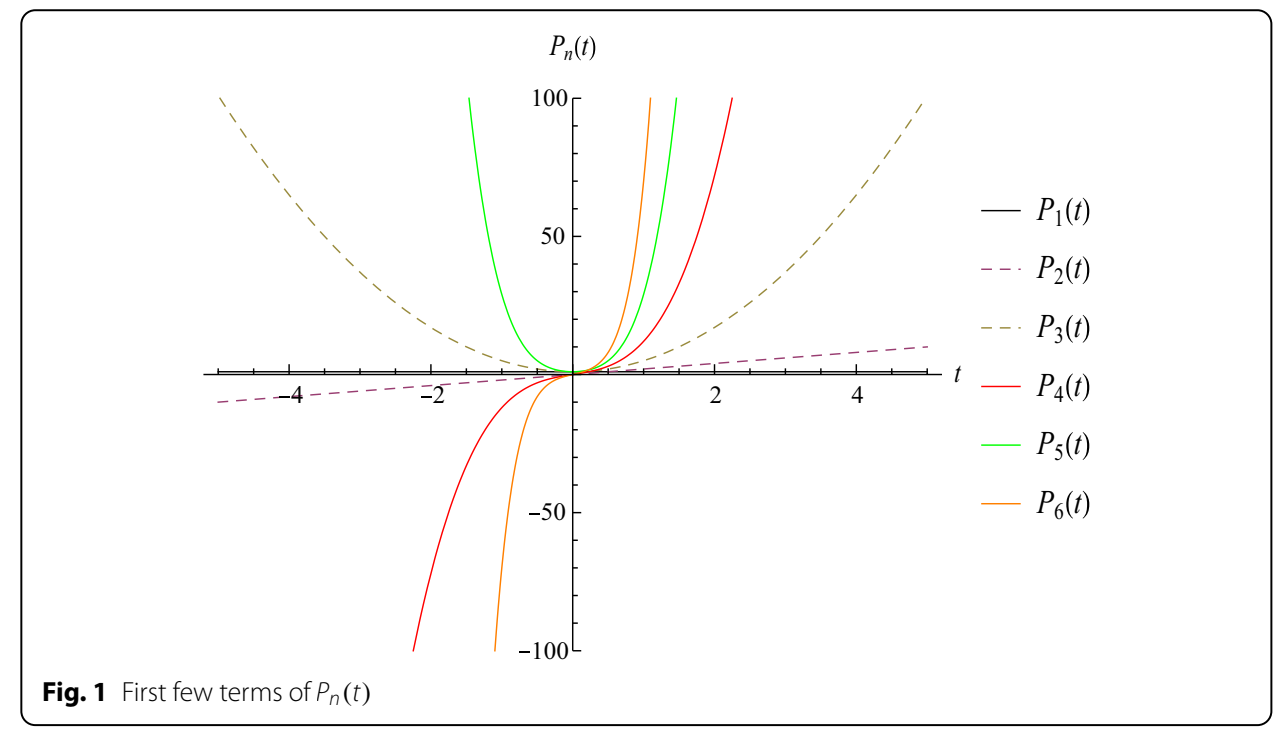


The present article is organized in the following manner: First, in "Preliminaries" section, the preliminaries regarding the properties of several polynomials, gamma and beta functions have been discussed to be used later in the subsequent sections. Then, the explicit formulas of some special orthogonal polynomials are given in "Methods" section. Apart from that, "Results and discussions" section includes some propositions and lemmas to be used later. In addition, some theorem have been proved regarding the sums of finite products of Pell polynomials in subsequent section. The final section is devoted for the concluding remarks.

\section{Preliminaries}

Definition 1 (Rising factorial polynomials and falling factorial polynomials) The rising factorial polynomials $\langle t\rangle_{n_{1}}$, for $n_{1} \geq 1$ are defined by [21]

$$
\langle t\rangle_{n_{1}}=t(t+1) \cdots\left(t+n_{1}-1\right)
$$

and the falling factorial polynomials $(t)_{n_{1}}$, for $n_{1} \geq 1$ are defined by [21]

$$
(t)_{n_{1}}=t(t-1) \cdots\left(t-n_{1}+1\right) .
$$

Furthermore, the rising factorial polynomials $\langle t\rangle_{n_{1}}$ and the falling factorial polynomials $(t)_{n_{1}}$ satisfy the following properties given by the following lemma.

\section{Lemma 1}

(a) For any nonnegative integer $n_{1},-\langle t\rangle_{n_{1}}=(-1)^{n_{1}}(t)_{n_{1}}$.

(b) For any nonnegative integer $n_{1},-(t)_{n_{1}}=(-1)^{n_{1}}\langle t\rangle_{n_{1}}$.

(c) The falling factorial polynomials $(t)_{n_{1}}$ can be written in terms of gamma function $\Gamma(t)$ as $(t)_{n_{1}}=\frac{\Gamma(t+1)}{\Gamma\left(t+1-n_{1}\right)}$, and similarly, the rising factorial polynomials can be represented by gamma function as $\langle t\rangle_{n_{1}}=\frac{\Gamma\left(t+n_{1}\right)}{\Gamma(t)}, n_{1} \geq 0$.

(d) For $n_{1} \geq n_{2} \geq 0, \frac{\left(2 n_{1}-2 n_{2}\right) !}{\left(n_{1}-n_{2}\right) !}=\frac{(-1)^{n_{2}}\left\langle\frac{1}{2}\right\rangle_{n_{1}} 2^{2 n_{1}-2 n_{2}}}{\left\langle\frac{1}{2}-n\right\rangle_{n_{2}}}$.

(e) For $n_{1} \geq 0, \Gamma\left(n_{1}+\frac{1}{2}\right)=\frac{\left(2 n_{1}\right) ! \sqrt{\pi}}{\left(n_{1}\right) ! 2^{2 n_{1}}}$.

\section{Proof}

The proof of the lemma can be referred from [21].

Definition 2 (Beta function) Now the beta function $B(x, y)$ is defined in terms of gamma function $\Gamma(x)$ as

$$
B(x, y)=\int_{0}^{1} t^{x-1}(1-t)^{y-1} d t=\frac{\Gamma(x) \Gamma(y)}{\Gamma(x+y)},
$$

for $\operatorname{Re}(x), \operatorname{Re}(y)>0$. 
Definition 3 (Hypergeometric function) The hypergeometric function is defined by [22]

$$
{ }_{m} F_{n}\left(a_{1}, \ldots, a_{m} ; b_{1}, \ldots, b_{n} ; t\right)=\sum_{s=0}^{\infty} \frac{\left\langle a_{1}\right\rangle_{s} \cdots\left\langle a_{m}\right\rangle_{s}}{\left\langle b_{1}\right\rangle_{s} \cdots\left\langle b_{n}\right\rangle_{s}} \frac{t^{s}}{s !},|t|<1, m \leq n+1 .
$$

Definition 4 (Gauss hypergeometric function and Chu-Vandermonde formula) A particular case of the hypergeometric function is the Gauss hypergeometric function defined by [23]

$$
{ }_{2} F_{1}\left(a_{1}, b_{1} ; c_{1} ; t\right)=\sum_{s=0}^{\infty} \frac{\left\langle a_{1}\right\rangle_{s}\left\langle b_{1}\right\rangle_{s}}{\left\langle c_{1}\right\rangle_{s}} \frac{t^{s}}{s !},|t|<1,
$$

and furthermore, a special case of Gauss hypergeometric function is the Chu-Vandermonde formula given by

$$
{ }_{2} F_{1}\left(-s, a_{1} ; c_{1} ; 1\right)=\frac{\left\langle c_{1}-a_{1}\right\rangle_{s}}{\left\langle c_{1}\right\rangle_{s}}, c_{1} \neq 0,-1, \cdots 1-s .
$$

Note: Furthermore, there is a link between the Pell polynomials $P_{n}(x)$ and Chebyshev polynomials of second kind denoted by $U_{n}(x)$. Before, proceeding to establish the connection, we need to define the Chebyshev polynomials of second kind.

Definition 5 (Chebyshev polynomials of second kind) The Chebyshev polynomials of second kind are given by the recurrence relation [24]

$$
U_{n}(x)=2 x U_{n-1}(x)-U_{n-2}(x), U_{0}(x)=1, U_{1}(x)=2 x, k \geq 2 .
$$

Hence, the first few terms of $U_{n}(x)$ are given by $U_{0}(x)=1, U_{1}(x)=2 x, U_{2}(x)=4 x^{2}-1$, $U_{3}(x)=8 x^{3}-4 x, U_{4}(x)=16 x^{4}-12 x^{2}+1, U_{5}(x)=32 x^{5}-32 x^{3}+6 x, \ldots$. The first few terms of $U_{n}(x)$ are graphically depicted in Fig. 2.

In addition, the Chebyshev polynomials are explicitly given by the formula, for $n \geq 0$,

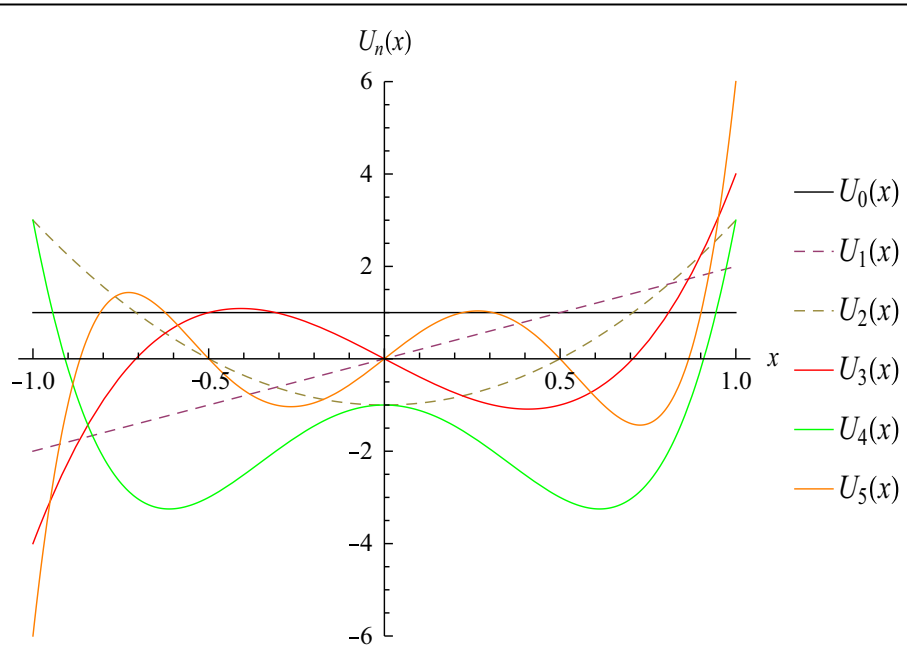

Fig. 2 First few terms of $U_{n}(x)$ 


$$
U_{n}(x)=(n+1)_{2} F_{1}\left(-n, 2+n ; \frac{3}{2} ; \frac{1-x}{2}\right)=\sum_{l=0}^{\left[\frac{n}{2}\right]}(-1)^{l}\left(\begin{array}{c}
n-l \\
l
\end{array}\right)(2 x)^{n-2 l}
$$

where ${ }_{2} F_{1}(a, b ; c ; x)$ can be referred from (13) and is given by the generating function

$$
\sum_{n=0}^{\infty} U_{n}(x) t^{n}=\frac{1}{1-2 x t+t^{2}}
$$

Lemma 2 The fundamental connection between the Chebyshev polynomials $U_{n}(x)$ of second kind and the Pell polynomials $P_{n}(x)$ is given by

$$
P_{n+1}(x)=\frac{1}{(\sqrt{-1})^{n}} U_{n}(\sqrt{-1} x) .
$$

\section{Proof}

The explicit expression for $P_{n+1}(x)$ can be referred from [5], given by

$$
P_{n+1}(x)=\sum_{l=0}^{\left[\frac{n}{2}\right]}\left(\begin{array}{c}
n-l \\
l
\end{array}\right)(2 x)^{n-2 l}, n \geq 0
$$

Hence, comparing (15) and (17), we get (16).

Note: The explicit expression for $P_{n+1}(x)$ can be either referred from [5] or can also be viewed by virtue of the combination of (16) and (15) after proving Lemma 2.

\section{Methods}

Proposition 1 Let $q(x) \in \mathbb{R}[x]$ be a polynomial of degree $n$ and further let $q(x)=\sum_{l=0}^{n} D_{l} P_{l+1}(x)$. Then,

$$
D_{l}=\frac{(l+1 !)(\sqrt{-1})^{l+1} 2^{l+1}}{(2 n+1) ! \pi} \int_{-\sqrt{-1}}^{\sqrt{-1}} q(x) \frac{d^{l}}{d x^{l}}\left(1+x^{2}\right)^{l+\frac{1}{2}} .
$$

\section{Proof}

By virtue of the orthogonality property of $U_{n}(x)$, we have

$$
\int_{-1}^{1}\left(1-x^{2}\right)^{\frac{1}{2}} U_{m}(x) U_{n}(x) d x=\frac{\pi \delta_{n, m}}{2}
$$

for $m, n \geq 0$. Combining (19) and the property (16), we have

$$
\int_{-\sqrt{-1}}^{\sqrt{-1}}\left(1+x^{2}\right)^{\frac{1}{2}} P_{m+1}(x) P_{n+1}(x) d x=\frac{\pi(\sqrt{-1})^{1-n-m} \delta_{n, m}}{2}
$$

which represents the orthogonality relation for the Pell polynomials $P_{n}(x)$. Furthermore, the Rodrigues' formula of the Chebyshev polynomials of second kind $U_{n}(x)$ is given, for $n \geq 0$, by 


$$
U_{n}(x)=\frac{(n+1 !)(-1)^{n} 2^{n}}{(2 n+1) !}\left(1-x^{2}\right)^{-\frac{1}{2}} \frac{d^{n}}{d x^{n}}\left(1-x^{2}\right)^{n+\frac{1}{2}}
$$

Again by combining (16) and (21), we get the Rodrigues' formula of the Pell polynomials as

$$
P_{n}(x)=\frac{(n+1 !) 2^{n}}{(2 n+1) !}\left(1+x^{2}\right)^{-\frac{1}{2}} \frac{d^{n}}{d x^{n}}\left(1+x^{2}\right)^{n+\frac{1}{2}}
$$

Now, by means of (20) and (22), we get the desired result.

\section{Results and discussions}

\section{Explicit formulas of special orthogonal polynomials}

In this section, some explicit definitions of certain polynomials have been recalled which will be used in the subsequent sections. The explicit formula for the Chebyshev polynomials of the first kind $\left(T_{n}(x)\right)$, those of the third kind $\left(V_{n}(x)\right)$, those of the fourth kind $\left(W_{n}(x)\right)$, Hermite polynomials $\left(H_{n}(x)\right)$, generalized Laguerre polynomials $\left(L_{n}^{\alpha}(x)\right)$, Legendre polynomials $\left(P_{n}(x)\right)$, Gegenbauer polynomials $\left(C_{n}^{(\lambda)}(x)\right)$, and Jacobi polynomials $\left(P_{n}^{(\alpha, \beta)}(x)\right)$. They are explicitly given by

$$
\begin{aligned}
& V_{n}(x)={ }_{2} F_{1}\left(-n, n+1 ; \frac{1}{2} ; \frac{1-x}{2}\right)=\sum_{l=0}^{n}\left(\begin{array}{c}
n+l \\
2 l
\end{array}\right) 2^{l}(x-1)^{l}, n \geq 0 \\
& W_{n}(x)=(2 n+1)_{2} F_{1}\left(-n, n+1 ; \frac{3}{2} ; \frac{1-x}{2}\right)=\sum_{l=0}^{n}\left(\begin{array}{c}
n+l \\
2 l
\end{array}\right) 2^{l}(x-1)^{l}, n \geq 0 \\
& T_{n}(x)=\frac{n}{2}{ }_{2} F_{1}\left(-n, n ; \frac{1}{2} ; \frac{1-x}{2}\right)=\sum_{l=0}^{\left[\frac{n}{2}\right]} \frac{(-1)^{l}}{n-l}\left(\begin{array}{c}
n-l \\
l
\end{array}\right)(2 x)^{n-2 l}, n \geq 1 \\
& H_{n}(x)=n ! \sum_{l=0}^{\left[\frac{n}{2}\right]} \frac{(-1)^{l}}{l !(n-l) !}(2 x)^{n-2 l}, n \geq 0 \\
& \mathcal{P}_{n}(x)={ }_{2} F_{1}\left(-n, n+1 ; 1 ; \frac{1-x}{2}\right)=\frac{1}{2^{n}} \sum_{l=0}^{\left[\frac{n}{2}\right]}(-1)^{l}\left(\begin{array}{c}
n \\
l
\end{array}\right)\left(\begin{array}{c}
2 n-2 l \\
n
\end{array}\right) x^{n-2 l}, n \geq 0 \\
& L_{n}^{\alpha}(x)=\frac{\langle\alpha+1\rangle_{n}}{n !}{ }_{1} F_{1}(-n ; \alpha+1 ; x)=\sum_{l=0}^{n} \frac{(-1)^{l}\left(\begin{array}{c}
n+\alpha \\
n-l
\end{array}\right)}{l !} x^{l}, n \geq 0, \alpha>-1
\end{aligned}
$$




$$
\begin{gathered}
\mathcal{P}_{n}^{\alpha, \beta}(x)=\frac{\langle\alpha+1\rangle_{n}}{n !}{ }_{2} F_{1}\left(-n, \alpha+\beta+n+1 ; \alpha+1 ; \frac{1-x}{2}\right) \\
=\sum_{k=0}^{n}\left(\begin{array}{c}
n+\alpha \\
n-k
\end{array}\right)\left(\begin{array}{c}
n+\beta \\
k
\end{array}\right)\left(\frac{x-1}{2}\right)^{k}\left(\frac{x+1}{2}\right)^{n-k}, \\
C_{n}^{\lambda}(x)=\left(\begin{array}{c}
n-1+2 \lambda \\
n
\end{array}\right){ }_{2} F_{1}\left(-n, 2 \lambda+n ; \lambda+\frac{1}{2} ; \frac{1-x}{2}\right) \\
=\sum_{k=0}^{\left[\frac{n}{2}\right]}(-1)^{k} \frac{\Gamma(n+\lambda-k)}{\gamma(\lambda)(n-2 k) ! k !}(2 x)^{n-2 k}, n \geq 0, \lambda>-\frac{1}{2}, \lambda \neq 0 .
\end{gathered}
$$

Proposition $2[1,2]$. Let $q(x) \in \mathbb{R}[x]$ be a polynomial of degree $n$. Then, we have the following:

(a) $q(x)=\sum_{k=0}^{n} C_{k, 1} W_{k}(x)$,

where

$$
C_{k, 1}=\frac{(-1)^{k} 2^{k} k !}{\pi(2 k) !} \int_{-1}^{1} q(x) \frac{d^{k}}{d x^{k}}(1+x)^{k-\frac{1}{2}}(1-x)^{k+\frac{1}{2}} d x .
$$

(b) $q(x)=\sum_{k=0}^{n} C_{k, 2} V_{k}(x)$,

where

$$
C_{k, 2}=\frac{(-1)^{k} 2^{k} k !}{\pi(2 k) !} \int_{-1}^{1} q(x) \frac{d^{k}}{d x^{k}}(1+x)^{k+\frac{1}{2}}(1-x)^{k-\frac{1}{2}} d x .
$$

(c) $q(x)=\sum_{k=0}^{n} C_{k, 3} T_{k}(x)$, where $C_{k, 3}=\frac{(-1)^{k} 2^{k} k ! \epsilon_{k}}{\pi(2 k) !} \int_{-1}^{1} q(x) \frac{d^{k}}{d x^{k}}\left(1-x^{2}\right)^{k-\frac{1}{2}} d x$.

(d) $q(x)=\sum_{k=0}^{n} C_{k, 4} U_{k}(x)$, where $C_{k, 4}=\frac{(-1)^{k} 2^{k+1}(k+1) !}{\pi(2 k+1) !} \int_{-1}^{1} q(x) \frac{d^{k}}{d x^{k}}\left(1-x^{2}\right)^{k+\frac{1}{2}} d x$.

(e) $q(x)=\sum_{k=0}^{n} C_{k, 5} H_{k}(x)$, where $C_{k, 5}=\frac{(-1)^{k}}{\sqrt{\pi} k ! 2^{k}} \int_{-\infty}^{\infty} q(x) \frac{d^{k}}{d x^{k}} e^{-x^{2}} d x$.

(f) $q(x)=\sum_{k=0}^{n} C_{k, 6} \mathcal{P}_{k}(x)$, where $C_{k, 6}=\frac{2 k+1}{k ! 2^{k+1}} \int_{-1}^{1} q(x) \frac{d^{k}}{d x^{k}}\left(x^{2}-1\right)^{k} d x$.

(g) $q(x)=\sum_{k=0}^{n} C_{k, 7} L_{k}^{\alpha}(x)$, where $C_{k, 7}=\frac{1}{\Gamma(\alpha+k+1)} \int_{0}^{\infty} q(x) \frac{d^{k}}{d x^{k}}\left(x^{\alpha+k} e^{-x}\right) d x$.

(h) $q(x)=\sum_{k=0}^{n} C_{k, 8} \mathcal{P}_{k}^{\alpha, \beta}(x) \quad$ where

$$
C_{k, 8}=\frac{(-1)^{k}(\alpha+\beta+2 k+1) \Gamma(\alpha+\beta+k+1)}{\Gamma(\alpha+k+1) \Gamma(\beta+k+1) 2^{\alpha+\beta+k+1}} \int_{-1}^{1} q(x) \frac{d^{k}}{d x^{k}}(1+x)^{k+\beta}(1-x)^{k+\alpha} d x .
$$

(i) $q(x)=\sum_{k=0}^{n} C_{k, 9} C_{k}^{\lambda}(x)$,

where

$$
C_{k, 9}=\frac{\Gamma(\lambda)(\lambda+k)}{\Gamma\left(\lambda+k+\frac{1}{2}\right)(-2)^{k} \sqrt{\pi}} \int_{-1}^{1} q(x) \frac{d^{k}}{d x^{k}}\left(1-x^{2}\right)^{k+\lambda-\frac{1}{2}} d x .
$$

Proposition 3 1, 2]. Let $m, k$ be nonnegative integers. Then, the following identities hold:

(i)

$$
\int_{-1}^{1} x^{m}\left(1-x^{2}\right)^{k-\frac{1}{2}} d x= \begin{cases}0, & m \equiv 1 \quad(\bmod 2) \\ \frac{m ! \pi(2 k) !}{2^{m+2 k}\left(\frac{m}{2}+k\right) !\left(\frac{m}{2}\right) ! k !}, & \text { if } m \equiv 0 \quad(\bmod 2)\end{cases}
$$


(ii)

$$
\int_{-1}^{1} x^{m}\left(1-x^{2}\right)^{k+\frac{1}{2}} d x= \begin{cases}0, & m \equiv 1 \quad(\bmod 2) ; \\ \frac{m ! \pi(2 k+2) !}{2^{m+2 k+2}\left(\frac{m}{2}+k+1\right) !\left(\frac{m}{2}\right) !(k+1) !}, & \text { if } m \equiv 0 \quad(\bmod 2) .\end{cases}
$$

(iii)

$\int_{-1}^{1} x^{m}(1+x)^{k+\frac{1}{2}}(1-x)^{k-\frac{1}{2}} d x= \begin{cases}\frac{(m+1) !(2 k) ! \pi}{2^{m+2 k+1}\left(\frac{m+1}{2}+k\right) !\left(\frac{m+1}{2}\right) !(k) !}, & m \equiv 1 \quad(\bmod 2) ; \\ \frac{m ! m(2 k) !}{2^{m+2 k}\left(\frac{m}{2}+k\right) !\left(\frac{m}{2}\right) !(k) !}, & \text { if } m \equiv 0 \quad(\bmod 2) .\end{cases}$

(iv)

$\int_{-1}^{1} x^{m}(1+x)^{k-\frac{1}{2}}(1-x)^{k+\frac{1}{2}} d x= \begin{cases}-\frac{(m+1) !(2 k) ! \pi}{2^{m+2 k+1}\left(\frac{m+1}{2}+k\right) !\left(\frac{m+1}{2}\right) !(k) !}, & m \equiv 1 \quad(\bmod 2) ; \\ \frac{m ! \pi(2 k) !}{2^{m+2 k}\left(\frac{m}{2}+k\right) !\left(\frac{m}{2}\right) !(k) !}, & \text { if } m \equiv 0 \quad(\bmod 2) .\end{cases}$

(v)

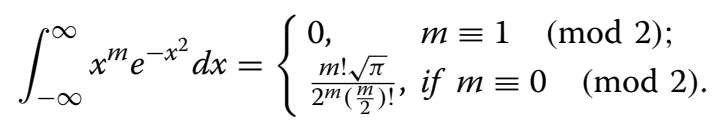

(vi)

$$
\int_{-1}^{1} x^{m}\left(1-x^{2}\right)^{k} d x= \begin{cases}0, & m \equiv 1 \quad(\bmod 2) \\ \frac{m ! k ! 2^{2 k+2}\left(\frac{m}{2}+k+1\right) !}{\left(\frac{m}{2}\right) !(m+2 k+2) !}, & \text { if } m \equiv 0 \quad(\bmod 2) .\end{cases}
$$

(vii)

$$
\int_{-1}^{1} x^{m}\left(1-x^{2}\right)^{k+\lambda-\frac{1}{2}} d x= \begin{cases}0, & m \equiv 1 \quad(\bmod 2) \\ \frac{\Gamma\left(k+\lambda+\frac{1}{2}\right) \Gamma\left(\frac{m+1}{2}\right)}{\Gamma\left(k+\lambda+1+\frac{m}{2}\right)}, & \text { if } m \equiv 0 \quad(\bmod 2) .\end{cases}
$$

$$
\int_{-1}^{1} x^{m}(1-x)^{k+\alpha}(1+x)^{k+\beta} d x=2^{\alpha+\beta+2 k+1} \sum_{s=0}^{m}\left(\begin{array}{c}
m \\
s
\end{array}\right)(-1)^{m-s} 2^{5} \frac{\Gamma(k+\beta+1+s) \Gamma(k+\alpha+1)}{\Gamma(\alpha+\beta+2 k+s+2)} .
$$

Lemma 3 For any nonnegative integers $n, k$, the following identity holds, for the summation of finite products of the Pell polynomial $P_{n}(x)$ over all nonnegative integers $j_{1}, j_{2}, \ldots, i_{r+1}$ with $j_{1}+j_{2}+\cdots+j_{r+1}=n$, given by

$$
\sum_{j_{1}+j_{2}+\cdots+j_{r+1}=n} P_{j_{1}+1}(x) P_{j_{2}+1}(x) \cdots P_{j_{r+1}+1}(x)=\frac{1}{r ! 2^{r}} P_{n+r+1}^{(r)}(x) .
$$

\section{Proof}

Let us denote the generating function for the Pell polynomials as $G(x, t)$ and by definition, 


$$
G(x, t)=\sum_{n=0}^{\infty} P_{n}(x) t^{n}=\frac{1}{1-2 x t-t^{2}} .
$$

Then, differentiating $G(x, t)$ with repect to $\mathrm{x}, \mathrm{r}$ times, we get

$$
\frac{\partial^{r} G(x, t)}{\partial x^{r}}=\frac{2^{r} t^{r} r !}{\left(1-2 x t-t^{2}\right)^{r+1}}
$$

from which we obtain

$$
\begin{aligned}
& \left(\frac{1}{1-2 x t-t^{2}}\right)^{r+1}=\frac{1}{2^{r} t^{r} r !} \frac{\partial^{r} G(x, t)}{\partial x^{r}} \\
& =\frac{1}{2^{r} r !} \sum_{n=r+1}^{\infty} P_{n}^{(r)}(x) t^{n} \\
& =\frac{1}{2^{r} r !} \sum_{n=0}^{\infty} P_{n+r+1}^{(r)}(x) t^{n+r+1} .
\end{aligned}
$$

Furthermore, we have

$$
\begin{aligned}
& \left(\frac{1}{1-2 x t-t^{2}}\right)^{r+1}=\sum_{n=0}^{\infty} P_{j_{1}+1}(x) t^{j_{1}+1} P_{j_{2}+1}(x) t^{j_{1}+1} \cdots P_{j_{r+1}+1}(x) t^{j_{r+1}+1} \\
& =\sum_{n}^{\infty} \sum_{j_{1}+j_{2}+\cdots+j_{r+1}=n} P_{j_{1}+1}(x) P_{j_{2}+1}(x) \cdots P_{j_{r+1}+1}(x) .
\end{aligned}
$$

By virtue of (33) and (36), we get the desired result. It can be also observed that Lemma 3 can be proved in a simple way. However, it was proved in Lemma 5 of [1] that

$$
\sum_{j_{1}+j_{2}+\cdots+j_{r+1}=n} U_{j_{1}}(x) U_{j_{2}}(x) \cdots U_{j_{r+1}}(x)=\frac{1}{r ! 2^{r}} U_{n+r}^{(r)}(x) .
$$

Hence, combining (16) with (38), we have the desired result of Lemma 3.

Theorem 1 Let $n, r$ be nonnegative integers, and let $\epsilon_{n}=2-\delta_{n, 0}$. Then, the summation of finite products of the Pell polynomials can be represented as

$$
\begin{aligned}
& \sum_{j_{1}+j_{2}+\cdots+j_{r+1}=n} P_{j_{1}+1}(x) P_{j_{2}+1}(x) \cdots P_{j_{r+1}+1}(x) \\
= & \frac{1}{(\sqrt{-1})^{n} r !} \sum_{i=0}^{n}\left(\begin{array}{c}
r+\left[\frac{i}{2}\right] \\
r
\end{array}\right)\left(n+r-\left[\frac{i}{2}\right]\right)_{r} V_{n-i}(\sqrt{-1} x) \\
= & \frac{1}{(\sqrt{-1})^{n} r !} \sum_{i=0}^{n}(-1)^{i}\left(\begin{array}{c}
r+\left[\frac{i}{2}\right] \\
r
\end{array}\right)\left(n+r-\left[\frac{i}{2}\right]\right)_{r} W_{n-i}(\sqrt{-1} x)
\end{aligned}
$$




$$
\begin{aligned}
= & \frac{1}{(\sqrt{-1})^{n} r !} \sum_{i=0}^{\left[\frac{n}{2}\right]} \epsilon_{n-2 i}\left(\begin{array}{c}
r+i \\
r
\end{array}\right)(n+r-i)_{r} T_{n-2 i}(\sqrt{-1} x) \\
= & \frac{(r+n) !}{(\sqrt{-1})^{n} r !} \sum_{i=0}^{\left[\frac{n}{2}\right]} \frac{1}{(n-2 i) ! i !}{ }_{1} F_{1}(-i ;-n-r ;-1) H_{n-i}(\sqrt{-1} x) \\
= & \frac{(r+n) !}{(\sqrt{-1})^{n} r !} \sum_{i=0}^{\left[\frac{n}{2}\right]} \frac{2 n+1-4 i}{i !(n-i+\sqrt{1} 2)_{n-i}}{ }_{2} F_{1}(-i, i-n-\sqrt{1} 2 ;-n-r ; 1) \mathcal{P}_{n-2 i}(\sqrt{-1} x) \\
= & \frac{\Gamma(n+\alpha+1) 2^{n}}{(\sqrt{-1})^{n} r !} \sum_{k=0}^{n} \frac{(-1)^{k}}{\Gamma(k+\alpha+1)} \sum_{l=0}^{\left[\frac{n-k}{2}\right]} \frac{\left(-\frac{1}{4}\right)^{l}(n-l+r) !}{l !(n-k-2 l) !(n+\alpha)_{2 l}} L_{k}^{(\alpha)}(\sqrt{-1} x) \\
= & \frac{(r+n) !}{(\sqrt{-1})^{n} r !(\lambda)_{n+1}} \sum_{i=0}^{\left[\frac{n}{2}\right]} \frac{(n-2 i+\lambda)(\lambda+n)_{i}}{2}{ }_{2} F_{1}(-i, i-\lambda-n ;-n-r ; 1) C_{n-i}^{(\lambda)}(\sqrt{-1} x) . \\
= & \frac{(-2)^{n}}{(\sqrt{-1})^{n} r !} \sum_{k=0}^{n} \frac{(-2)^{k} \Gamma(\alpha+\beta+k+1)}{\Gamma(\alpha+\beta+2 k+1)} \sum_{l=0}^{\left[\frac{n-k}{2}\right]} \frac{\left(-\frac{1}{4}\right)^{l}(n-l+r) !}{l !(n-k-2 l) !} \\
& \\
& \\
&
\end{aligned}
$$

\section{Proof}

The proof of the theorem is by virtue of Theorem 1 of [1], Theorem 1 of [2] and (16).

\section{Remark}

The $r$-th derivative of the Pell polynomial $P_{n+1}(x)$ is given by

$$
P_{n+1}^{(r)}=\sum_{l=0}^{\left[\frac{n-r}{2}\right]}\left(\begin{array}{c}
n-l \\
l
\end{array}\right)(n-2 l)_{r} 2^{n-2 l} x^{n-r-2 l} .
$$

Furthermore,

$$
P_{n+r+1}^{(r+k)}=\sum_{l=0}^{\left[\frac{n-k}{2}\right]}\left(\begin{array}{c}
n-l+r \\
l
\end{array}\right)(n+r-2 l)_{r+k} 2^{n+r-2 l} x^{n-k-2 l} .
$$

By default, we assume that

$$
\sum_{j_{1}+j_{2}+\cdots+j_{r+1}=n} P_{j_{1}+1}(x) P_{j_{2}+1}(x) \cdots P_{j_{r+1}+1}(x)=\eta_{n, r}(x)
$$

throughout the remaining part of the paper. 
Theorem 2 Let $n, r$ be nonnegative integers. Then, we obtain the following representations for the summation of finite products of the Pell polynomials

$$
\begin{aligned}
& \sum_{j_{1}+j_{2}+\cdots+j_{r+1}=n} P_{j_{1}+1}(x) P_{j_{2}+1}(x) \cdots P_{j_{r+1}+1}(x) \\
& =\left(\begin{array}{c}
r+n \\
n
\end{array}\right) \sum_{i=0}^{n}(-1)^{j}\left(\begin{array}{c}
n \\
{\left[\frac{i}{2}\right]}
\end{array}\right){ }_{2} F_{1}\left(-\left[\frac{i}{2}\right],\left[\begin{array}{c}
i \\
2
\end{array}\right]-n ;-n-r ;-1\right) W_{n-i}(x) \\
& \left.=\frac{(r+n) !}{n ! r !} \sum_{i=0}^{n}\left(\begin{array}{c}
n \\
{\left[\frac{i}{2}\right]}
\end{array}\right]\right){ }_{2} F_{1}\left(-\left[\frac{i}{2}\right],\left[\frac{i}{2}\right]-n ;-n-r ;-1\right) V_{n-i}(x) \\
& =\frac{(r+n) !}{r !} \sum_{i=0}^{\left[\frac{n}{2}\right]} \frac{\epsilon_{n-2 j}}{(n-i) ! i !} 2 F_{1}(-i, i-n ;-n-r ;-1) T_{n-2 i}(x) \\
& =\frac{(r+n) !}{r !} \sum_{i=0}^{\left[\frac{n}{2}\right]} \frac{n-2 i+1}{(n-i+1) ! i !}{ }^{2} F_{1}(-i, i-n-1 ;-n-r ;-1) U_{n-2 i}(x) \\
& =\frac{(r+n) !}{r !} \sum_{i=0}^{n} \frac{1}{(n-2 i) ! i !}{ }_{1} F_{1}(-i ;-n-r ; 1) H_{n-i}(x) \\
& =\frac{(r+n) !}{r !} \sum_{i=0}^{\left[\frac{n}{2}\right]} \frac{2 n+1-4 i}{i !\left(n-i+\frac{1}{2}\right)_{n-i}}{ }_{2} F_{1}\left(-i, i-n-\frac{1}{2} ;-n-r ;-1\right) \mathcal{P}_{n-2 i}(x) \\
& =\frac{2^{n}}{r !} \Gamma(n+1+\alpha) \sum_{k=0}^{n} \frac{(-1)^{k}}{\Gamma(k+\alpha+1)} \sum_{l=0}^{\left[\frac{n-k}{2}\right]} \frac{\left(-\frac{1}{4}\right)^{l}(n-l+r) !}{l !(n-k-2 l) !(n+\alpha)_{2 l}} L_{k}^{\alpha}(x) \\
& =\frac{(-2)^{n}}{r !} \sum_{k=0}^{n} \frac{(-2)^{k} \Gamma(\alpha+\beta+k+1)}{\Gamma(\alpha+\beta+2 k+1)} \sum_{l=0}^{\left[\frac{n-k}{2}\right]} \frac{\left(-\frac{1}{4}\right)^{l}(n-l+r) !}{l !(n-k-2 l) !} \\
& \times{ }_{2} F_{1}(2 l+k-n, \beta+k+1 ; \alpha+\beta+2 k+2 ; 2) \mathcal{P}_{k}^{(\alpha, \beta)}(x) \\
& =\frac{(r+n) !}{r !(\lambda)_{n+1}} \sum_{i=0}^{\left[\frac{n}{2}\right]} \frac{(n-2 i+\lambda)(\lambda+n)_{i}}{i !}{ }_{2} F_{1}(-i, i-\lambda-n ;-n-r ;-1) C_{n-2 i}^{(\lambda)}(x) .
\end{aligned}
$$

\section{Proof}

In order to prove (49), we let

$$
\eta_{n, r}(x)=\sum_{k=0}^{n} C_{k, 1} W_{k}(x)
$$


Then, by virtue of Proposition 2(a), we obtain

$$
C_{k, 1}=\frac{(-1)^{k} 2^{k} k !}{\pi(2 k) !} \int_{-1}^{1} \eta_{n, r}(x) \frac{d^{k}}{d x^{k}}(1+x)^{k-\frac{1}{2}}(1-x)^{k+\frac{1}{2}} d x .
$$

Then, by (31) and (48) and subsequently integration by parts $k$ times, we get

$$
\begin{aligned}
C_{k, 1}= & \frac{(-1)^{k} 2^{k} k !}{\pi(2 k) !} \int_{-1}^{1} \eta_{n, r}(x) \frac{d^{k}}{d x^{k}}(1+x)^{k-\frac{1}{2}}(1-x)^{k+\frac{1}{2}} d x \\
= & \frac{(-1)^{k} 2^{k} k !}{\pi(2 k) ! 2^{r} r !} \int_{-1}^{1} P_{n+r+1}^{(r)}(x) \frac{d^{k}}{d x^{k}}(1+x)^{k-\frac{1}{2}}(1-x)^{k+\frac{1}{2}} d x \\
= & \frac{2^{k} k !}{\pi(2 k) ! 2^{r} r !} \int_{-1}^{1} P_{n+r+1}^{(r+k)}(x)(1+x)^{k-\frac{1}{2}}(1-x)^{k+\frac{1}{2}} d x \\
= & \frac{2^{k} k !}{\pi(2 k) ! 2^{r} r !} \sum_{l=0}^{\left[\frac{n-k}{2}\right]}\left(\begin{array}{c}
n-l+r \\
l
\end{array}\right)(n+r-2 l)_{r+k} 2^{n+r-2 l} \times \\
& \int_{-1}^{1} x^{n-k-2 l}(1+x)^{k-\frac{1}{2}}(1-x)^{k+\frac{1}{2}} d x .
\end{aligned}
$$

Now, by virtue of Proposition 3(iv), we have

$$
\int_{-1}^{1} x^{n-k-2 l}(1+x)^{k-\frac{1}{2}}(1-x)^{k+\frac{1}{2}} d x=\left\{\begin{array}{lll}
-\frac{(n-k-2 l+1) !(2 k) ! \pi}{2^{n+k-2 l+1}\left(\frac{n+k-2 l+1}{2}\right) !\left(\frac{n-k-2 l+1}{2}\right) !(k) !}, & n \neq \equiv k & (\bmod 2) ; \\
\frac{(n-k-2 l) !(2 k) ! \pi}{2^{n+k-2 l}\left(\frac{n+k-2 l}{2}\right) !\left(\frac{n-k-2 l}{2}\right) !(k) !}, & \text { if } n \equiv k \quad(\bmod 2) .
\end{array}\right.
$$

After further simplifying (58)-(60), we get

$$
\begin{aligned}
\eta_{n, r}(x)=-\frac{1}{r !} \sum_{\substack{k=0 \\
n \neq k(\bmod 2)}}^{\sum_{l=0}^{n}} \frac{(n-k+1-2 l)(n-l+r) !}{2 l !\left(\frac{n+k+1}{2}-l\right) !\left(\frac{n-k+1}{2}-l\right) !} W_{k}(x) \\
+\frac{1}{r !} \sum_{\substack{k=0 \\
n \equiv k}}^{\substack{\left.\frac{n-k}{2}\right] \\
(\bmod 2)}}
\end{aligned}
$$

Now, let us substitute $n-2 i-1$ in place of $k$ in the first summation term and $n-2 i$ in place of $k$ in the second summation term which results in

$$
\begin{aligned}
\eta_{n, r}(x) & =\frac{1}{r !} \sum_{i=0}^{\left[\frac{n}{2}\right]} \sum_{l=0}^{i} \frac{(n-l+r) !}{(n-l-i) !(i-l) ! l !} W_{n-2 i}(x) \\
& -\frac{1}{r !} \sum_{i=0}^{\left[\frac{n-1}{2}\right]} \sum_{l=0}^{i} \frac{(n-l+r) !}{(n-l-i) !(i-l) ! l !} W_{n-2 i-1}(x) .
\end{aligned}
$$

Furthermore, by virtue of the rising factorial polynomials and the falling factorial polynomials in (10), (11) and their properties in 1(a) and (b), we can write 


$$
\begin{aligned}
\eta_{n, r}(x)= & \left(\begin{array}{c}
r+n \\
n
\end{array}\right) \sum_{i=0}^{\left[\frac{n}{2}\right]}\left(\begin{array}{c}
n \\
i
\end{array}\right) \sum_{l=0}^{i} \frac{\langle-i\rangle_{l}\langle i-n\rangle_{l}}{\langle-n-r\rangle_{l}} \frac{(-1)^{l}}{l !} W_{n-2 i}(x) \\
& -\left(\begin{array}{c}
r+n \\
n
\end{array}\right) \sum_{i=0}^{\left[\frac{n-1}{2}\right]}\left(\begin{array}{c}
n \\
i
\end{array}\right) \sum_{l=0}^{i} \frac{\langle-i\rangle_{l}\langle i-n\rangle_{l}}{\langle-n-r\rangle_{l}} \frac{(-1)^{l}}{l !} W_{n-2 i-1}(x) .
\end{aligned}
$$

A further simplification in terms of the hypergeometric function gives us

$$
\begin{aligned}
\eta_{n, r}(x) & =\left(\begin{array}{c}
r+n \\
n
\end{array}\right) \sum_{i=0}^{\left[\frac{n}{2}\right]}\left(\begin{array}{c}
n \\
i
\end{array}\right){ }_{2} F_{1}(-i, i-n ;-n-r ;-1) W_{n-2 i}(x) \\
& -\left(\begin{array}{c}
r+n \\
n
\end{array}\right) \sum_{i=0}^{\left[\frac{n-1}{2}\right]}\left(\begin{array}{c}
n \\
i
\end{array}\right){ }_{2} F_{1}(-i, i-n ;-n-r ;-1) W_{n-2 i-1}(x) \\
& \left.=\left(\begin{array}{c}
r+n \\
n
\end{array}\right) \sum_{i=0}^{n}(-1)^{i}\left(\begin{array}{c}
n \\
{\left[\frac{i}{2}\right.}
\end{array}\right]\right){ }_{2} F_{1}\left(-\left[\frac{i}{2}\right],\left[\frac{i}{2}\right]-n ;-n-r ;-1\right) W_{n-2 i}(x)
\end{aligned}
$$

which gives the desired result. The proof of (50) is similar to that of (49). Now, for proving (51), we proceed as in (49) and by taking

$$
\eta_{n, r}(x)=\sum_{k=0}^{n} C_{k, 3} T_{k}(x)
$$

Then, by virtue of Proposition 2(c), we obtain

$$
C_{k, 3}=\frac{(-1)^{k} 2^{k} k ! \epsilon_{k}}{\pi(2 k) !} \int_{-1}^{1} \eta_{n, r}(x) \frac{d^{k}}{d x^{k}}\left(1-x^{2}\right)^{k-\frac{1}{2}} d x .
$$

Then, by (31) and (48) and then using integration by parts $k$ times, we get

$$
\begin{aligned}
C_{k, 1}= & \frac{(-1)^{k} 2^{k} k ! \epsilon_{k}}{\pi(2 k) !} \int_{-1}^{1} \eta_{n, r}(x) \frac{d^{k}}{d x^{k}}\left(1-x^{2}\right)^{k-\frac{1}{2}} d x \\
= & \frac{(-1)^{k} 2^{k} k ! \epsilon_{k}}{\pi(2 k) ! 2^{r} r !} \int_{-1}^{1} P_{n+r+1}^{(r)}(x) \frac{d^{k}}{d x^{k}}\left(1-x^{2}\right)^{k-\frac{1}{2}} d x \\
= & \frac{2^{k} k ! \epsilon_{k}}{\pi(2 k) ! 2^{r} r !} \int_{-1}^{1} P_{n+r+1}^{(r+k)}(x)\left(1-x^{2}\right)^{k-\frac{1}{2}} d x \\
= & \frac{2^{k} k ! \epsilon_{k}}{\pi(2 k) ! 2^{r} r !} \sum_{l=0}^{\left[\frac{n-k}{2}\right]}\left(\begin{array}{c}
n-l+r \\
l
\end{array}\right)(n+r-2 l)_{r+k} 2^{n+r-2 l} \\
& \times \int_{-1}^{1} x^{n-k-2 l}\left(1-x^{2}\right)^{k-\frac{1}{2}} d x .
\end{aligned}
$$

Now, by virtue of Proposition 3(i), we have

$$
\int_{-1}^{1} x^{n-k-2 l}\left(1-x^{2}\right)^{k-\frac{1}{2}} d x= \begin{cases}0, & n \neq k \quad(\bmod 2) \\ \frac{(n-k-2 l) ! \pi(2 k) !}{2^{n+k-2 l}\left(\frac{n+k}{2}-l\right) !\left(\frac{n-k}{2}-l\right) ! k !}, & \text { if } n \equiv k \quad(\bmod 2) .\end{cases}
$$

Furthermore, using (61)-(63), and after some simplifications, we obtain 


$$
\eta_{n, r}(x)=\frac{1}{r !} \sum_{\substack{k=0 \\ n \equiv k}}^{n} \epsilon_{k} \sum_{l=0}^{\left[\frac{n-k}{2}\right]} \frac{(n-l+r) !}{l !\left(\frac{n+k}{2}-l\right) !\left(\frac{n-k}{2}-l\right) !} T_{k}(x) .
$$

Then, putting $k=n-2 i$ and using (10), (11), we have

$$
\begin{aligned}
\eta_{n, r}(x) & =\frac{1}{r !} \sum_{i=0}^{\left[\frac{n}{2}\right]} \epsilon_{n-2 i} \sum_{l=0}^{i} \frac{(n-l+r) !}{(n-l-i) !(i-l) ! l !} T_{n-2 i}(x) \\
& =\frac{(r+n) !}{r !} \sum_{i=0}^{\left[\frac{n}{2}\right]} \frac{\epsilon_{n-2 i}}{(n-i) ! i !} \sum_{l=0}^{i} \frac{\langle-i\rangle_{l}\langle i-n\rangle_{l}}{\langle-n-r\rangle_{l}} \frac{(-1)^{l}}{l !} T_{n-2 i}(x),
\end{aligned}
$$

which on further simplification lends us

$$
\eta_{n, r}(x)=\frac{(r+n) !}{r !} \sum_{i=0}^{\left[\frac{n}{2}\right]} \frac{\epsilon_{n-2 i}}{(n-i) ! i !}{ }^{2} F_{1}(-i, i-n ;-n-r ;-1) T_{n-2 i}(x) .
$$

Similarly for proving (52), let us assume

$$
\eta_{n, r}(x)=\sum_{k=0}^{n} C_{k, 4} U_{k}(x) .
$$

Then, by virtue of Proposition 2(d), we obtain

$$
C_{k, 4}=\frac{(-1)^{k} 2^{k+1}(k+1) !}{\pi(2 k+1) !} \int_{-1}^{1} \eta_{n, r}(x) \frac{d^{k}}{d x^{k}}\left(1-x^{2}\right)^{k+\frac{1}{2}} d x .
$$

Then, by (31) and (48) and then using integration by parts $k$ times, we get

$$
\begin{aligned}
C_{k, 4}= & \frac{2^{k} k ! \epsilon_{k}}{\pi(2 k) ! 2^{r} r !} \int_{-1}^{1} P_{n+r+1}^{(r+k)}(x)\left(1-x^{2}\right)^{k+\frac{1}{2}} d x \\
= & \frac{2^{k} k ! \epsilon_{k}}{\pi(2 k) ! 2^{r} r !} \sum_{l=0}^{\left[\frac{n-k}{2}\right]}\left(\begin{array}{c}
n-l+r \\
l
\end{array}\right)(n+r-2 l)_{r+k} 2^{n+r-2 l} \times \\
& \int_{-1}^{1} x^{n-k-2 l}\left(1-x^{2}\right)^{k+\frac{1}{2}} d x .
\end{aligned}
$$

Now, by virtue of Proposition 3(ii), we have

$$
\int_{-1}^{1} x^{n-k-2 l}\left(1-x^{2}\right)^{k+\frac{1}{2}} d x= \begin{cases}0, & n \neq k \quad(\bmod 2) ; \\ \frac{(n-k-2 l) ! \pi(2 k+2) !}{2^{n+k-2 l+2}\left(\frac{n+k}{2}-l+1\right) !\left(\frac{n-k}{2}-l\right) !(k+1) !}, & \text { if } n \equiv k \quad(\bmod 2) .\end{cases}
$$

Furthermore, using (64)-(66), and after some simplification, we obtain 


$$
\eta_{n, r}(x)=\frac{1}{r !} \sum_{\substack{k=0 \\ n \equiv k}}^{n}(k+1) \sum_{l=0}^{\left[\frac{n-k}{2}\right]} \frac{(n-l+r) !}{l !\left(\frac{n+k}{2}-l+1\right) !\left(\frac{n-k}{2}-l\right) !} U_{k}(x) .
$$

Then, similar to the previous cases, putting $k=n-2 i$ and using (10), (11), we have

$$
\begin{aligned}
\eta_{n, r}(x) & =\frac{1}{r !} \sum_{i=0}^{\left[\frac{n}{2}\right]}(n-2 i+1) \sum_{l=0}^{i} \frac{(n-l+r) !}{(n-l-i+1) !(i-l) ! l !} U_{n-2 i}(x) \\
& =\frac{(r+n) !}{r !} \sum_{i=0}^{\left[\frac{n}{2}\right]} \frac{n-2 i+1}{(n-i+1) ! i !} \sum_{l=0}^{i} \frac{\langle-i\rangle_{l}\langle i-n-1\rangle_{l}}{\langle-n-r\rangle_{l}} \frac{(-1)^{l}}{l !} U_{n-2 i}(x),
\end{aligned}
$$

which on further simplification, as in the previous cases, will give us the desired result. A similar procedure can be followed for the proof of (53). Next, for (54), we let $\eta_{n, r}(x)=\sum_{k=0}^{n} C_{k, 6} \mathcal{P}_{k}(x)$. Then, by virtue of Proposition 2(f), we obtain

$$
C_{k, 6}=\frac{2 k+1}{k ! 2^{k+1}} \int_{-1}^{1} \eta_{n, r}(x) \frac{d^{k}}{d x^{k}}\left(x^{2}-1\right)^{k} d x .
$$

Then, by (31) and (48) and then using integration by parts $k$ times, we get

$$
\begin{aligned}
& C_{k, 4}=\frac{2 k+1}{k ! 2^{k+1} 2^{r} r !} \int_{-1}^{1} P_{n+r+1}^{(r+k)}(x)\left(1-x^{2}\right)^{k} d x \\
& =\frac{2 k+1}{k ! 2^{k+1} 2^{r} r !} \sum_{l=0}^{\left[\frac{n-k}{2}\right]}\left(\begin{array}{c}
n-l+r \\
l
\end{array}\right)(n+r-2 l)_{r+k} 2^{n+r-2 l} \int_{-1}^{1} x^{n-k-2 l}\left(1-x^{2}\right)^{k} d x .
\end{aligned}
$$

Now, by virtue of Proposition 3(vi), we have

$$
\int_{-1}^{1} x^{n-k-2 l}\left(1-x^{2}\right)^{k} d x= \begin{cases}0, & n \not \equiv k \quad(\bmod 2) \\ \frac{(n-k-2 l) ! k ! 2^{2 k+2}\left(\frac{n+k}{2}-l+1\right) !}{\left(\frac{n-k}{2}-l\right) !(n+k-2 l+2) !}, & \text { if } n \equiv k \quad(\bmod 2) .\end{cases}
$$

After some modifications and putting $k=n-2 i$ and further using (10), (11), we have

$$
\eta_{n, r}(x)=\frac{1}{r !} \sum_{i=0}^{\left[\frac{n}{2}\right]}(2 n-4 i+1) \sum_{l=0}^{i} \frac{(n-l+r) !(n-l-i+1) ! 2^{(2 n-2 l-2 i+1)}}{(2 n-2 l-2 i+2) !(i-l) ! l !} \mathcal{P}_{n-2 i}(x) .
$$

Then, by virtue of $1(\mathrm{~d})$ and (10)

$$
\eta_{n, r}(x)=\frac{(r+n) !}{r !} \sum_{i=0}^{\left[\frac{n}{2}\right]} \frac{2 n-4 i+1}{\left(n-i+\frac{1}{2}\right)_{n-i} ! i !} \sum_{l=0}^{i} \frac{\langle-i\rangle_{l}\left\langle i-n-\frac{1}{2}\right\rangle_{l}}{\langle-n-r\rangle_{l}} \frac{(-1)^{l}}{l !} \mathcal{P}_{n-2 i}(x),
$$

which on further simplification, as in the previous cases, will give us the desired result. Next, to prove (55), assuming 


$$
\eta_{n, r}(x)=\sum_{k=0}^{n} C_{k, 7} L_{k}(x)
$$

Then, by virtue of Proposition 2(g), we obtain

$$
C_{k, 7}=\frac{1}{\Gamma(\alpha+k+1)} \int_{0}^{\infty} \eta_{n, r}(x) \frac{d^{k}}{d x^{k}}\left(x^{\alpha+k} e^{-x}\right) d x
$$

Then, by (31) and (48) and using integration by parts $k$ times, we get

$$
\begin{aligned}
C_{k, 7}= & \frac{(-1)^{k}}{\Gamma(\alpha+k+1) 2^{r} r !} \int_{0}^{\infty} P_{n+r+1}^{(r+k)}(x) x^{k+\alpha} e^{-x} d x \\
= & \frac{(-1)^{k}}{\Gamma(\alpha+k+1) 2^{r} r !} \sum_{l=0}^{\left[\frac{n-k}{2}\right]}\left(\begin{array}{c}
n-l+r \\
l
\end{array}\right)(n+r-2 l)_{r+k} 2^{n+r-2 l} \\
& \times \int_{0}^{\infty} x^{n+\alpha-2 l} e^{-x} d x .
\end{aligned}
$$

After some modifications and putting $k=n-2 i$ and further using (10), (11), we have

$$
\eta_{n, r}(x)=\frac{2^{n}}{r !} \sum_{k=0}^{n} \frac{(-1)^{k}}{\Gamma(\alpha+k+1)} \sum_{l=0}^{\left[\frac{n-k}{2}\right]} \frac{\left(\frac{1}{4}\right)^{l}(n-l+r) ! \Gamma(n+\alpha-2 l+1)}{l !} L_{k}^{\alpha}(x) .
$$

Then, by virtue of Lemma 1(c), we get

$$
\eta_{n, r}(x)=\frac{2^{n} \Gamma(\alpha+n+1)}{r !} \sum_{k=0}^{n} \frac{(-1)^{k}}{\Gamma(\alpha+k+1)} \sum_{l=0}^{\left[\frac{n-k}{2}\right]} \frac{\left(\frac{1}{4}\right)^{l}(n-l+r) !}{(n+\alpha)_{2 l}(n-k-2 l) ! l !} L_{k}^{\alpha}(x) .
$$

Now, for (56), we proceed as in (55) and by taking

$$
\eta_{n, r}(x)=\sum_{k=0}^{n} C_{k, 8} \mathcal{P}_{k}^{(\alpha, \beta)}(x)
$$

Then, by virtue of Proposition 2(h), we obtain

$$
C_{k, 8}=\frac{(-1)^{k}(\alpha+\beta+2 k+1) \Gamma(\alpha+\beta+k+1)}{\Gamma(\alpha+k+1) \Gamma(\beta+k+1) 2^{\alpha+\beta+k+1}} \int_{-1}^{1} \eta_{n, r}(x) \frac{d^{k}}{d x^{k}}(1+x)^{k+\beta}(1-x)^{k+\alpha} .
$$

Then, by (31) and (48) and then using integration by parts $k$ times, we get 


$$
\begin{aligned}
C_{k, 8}= & \frac{(-1)^{k}(\alpha+\beta+2 k+1) \Gamma(\alpha+\beta+k+1)}{\Gamma(\alpha+k+1) \Gamma(\beta+k+1) 2^{\alpha+\beta+k+1}} \int_{-1}^{1} \eta_{n, r}(x) \frac{d^{k}}{d x^{k}}(1+x)^{k+\beta}(1-x)^{k+\alpha} d x \\
= & \frac{(-1)^{k}(\alpha+\beta+2 k+1) \Gamma(\alpha+\beta+k+1)}{\Gamma(\alpha+k+1) \Gamma(\beta+k+1) 2^{\alpha+\beta+k+1} 2^{r} r !} \int_{-1}^{1} P_{n+r+1}^{(r)}(x) \frac{d^{k}}{d x^{k}}(1+x)^{k+\beta}(1-x)^{k+\alpha} d x \\
= & \frac{(\alpha+\beta+2 k+1) \Gamma(\alpha+\beta+k+1)}{\Gamma(\alpha+k+1) \Gamma(\beta+k+1) 2^{\alpha+\beta+k+1} 2^{r} r !} \int_{-1}^{1} P_{n+r+1}^{(r+k)}(x)(1+x)^{k+\beta}(1-x)^{k+\alpha} d x \\
= & \frac{(\alpha+\beta+2 k+1) \Gamma(\alpha+\beta+k+1)}{\Gamma(\alpha+k+1) \Gamma(\beta+k+1) 2^{\alpha+\beta+k+1} 2^{r} r !} \\
& \times \sum_{l=0}^{\left[\frac{n-k}{2}\right]}\left(\begin{array}{c}
n-l+r \\
l
\end{array}\right)(n+r-2 l)_{r+k} 2^{n+r-2 l} \\
& \times \int_{-1}^{1} x^{n-k-2 l}(1+x)^{k+\beta}(1-x)^{k+\alpha} d x .
\end{aligned}
$$

Now, by virtue of Proposition 3(viii), we have

$$
\begin{aligned}
& \int_{-1}^{1} x^{n-k-2 l}(1-x)^{k+\alpha}(1+x)^{k+\beta} d x=2^{\alpha+\beta+2 k+1} \times \\
& \sum_{s=0}^{n-k-2 l}\left(\begin{array}{c}
n-k-2 l \\
s
\end{array}\right)(-1)^{n-k-2 l-s} 2^{s} \frac{\Gamma(k+\beta+1+s) \Gamma(k+\alpha+1)}{\Gamma(\alpha+\beta+2 k+s+2)} .
\end{aligned}
$$

Furthermore, using (71)-(73), and after some simplifications, weobtain

$$
\begin{aligned}
\eta_{n, r}(x) & =\sum_{k=0}^{n} \frac{2^{\alpha+\beta+2 k+1}(\alpha+\beta+2 k+1) \Gamma(\alpha+\beta+k+1)}{\Gamma(\alpha+k+1) \Gamma(\beta+k+1) 2^{\alpha+\beta+k+1} 2^{r} r !} \sum_{l=0}^{\left[\frac{n-k}{2}\right]} \frac{\left(\frac{1}{4}\right)^{l}(n-l+r) !}{(n-k-l) ! l !} \\
& \sum_{s=0}^{n-k-2 l}\left(\begin{array}{c}
n-k-2 l \\
s
\end{array}\right)(-1)^{n-k-2 l-s} 2^{s} \frac{\Gamma(k+\beta+1+s) \Gamma(k+\alpha+1)}{\Gamma(\alpha+\beta+2 k+s+2)} \mathcal{P}_{k}^{(\alpha, \beta)}(x) .
\end{aligned}
$$

Then, using (10), (11), we have

$$
\begin{aligned}
\eta_{n, r}(x) & =\frac{(-2)^{n}}{r} \sum_{k=0}^{n} \frac{(-2)^{k} \Gamma(\alpha+\beta+k+1)}{\Gamma(\alpha+\beta+2 k+1)} \sum_{l=0}^{\left[\frac{n-k}{2}\right]} \frac{\left(\frac{1}{4}\right)^{l}(n-l+r) !}{(n-k-l) ! l !} \\
& \sum_{s=0}^{n-k-2 l} \frac{\langle k-n+2 l\rangle_{s}\langle k+\beta+1\rangle_{s}}{\langle\alpha+\beta+2 k+2\rangle_{s}} \frac{2^{s}}{s !} \mathcal{P}_{k}^{(\alpha, \beta)}(x)
\end{aligned}
$$

which on further simplification using the hypergeometric function in (12) will result in the identity (56). For the final proof of (57), we proceed similarly by assuming $\eta_{n, r}(x)=\sum_{k=0}^{n} C_{k, 9} C_{k}^{(\lambda)}(x)$. Then, by virtue of Proposition 2(i), we obtain

$$
C_{k, 9}=\frac{\Gamma(\lambda)(\lambda+k)}{\Gamma\left(\lambda+k+\frac{1}{2}\right)(-2)^{k} \sqrt{\pi} 2^{r} r !} \int_{-1}^{1} \eta_{n, r}(x) \frac{d^{k}}{d x^{k}}\left(1-x^{2}\right)^{k+\lambda-\frac{1}{2}} d x .
$$

Then, by applying (31) and (48) and further integrating by parts $k$ times, we get 


$$
\begin{aligned}
C_{k, 9}= & \frac{\Gamma(\lambda)(\lambda+k)}{\Gamma\left(\lambda+k+\frac{1}{2}\right) 2^{k} \sqrt{\pi} 2^{r} r !} \int_{-1}^{1} P_{n+r+1}^{(r+k)}(x)\left(1-x^{2}\right)^{k+\lambda-\frac{1}{2}} d x \\
= & \frac{\Gamma(\lambda)(\lambda+k)}{\Gamma\left(\lambda+k+\frac{1}{2}\right) 2^{k} \sqrt{\pi} 2^{r} r !} \sum_{l=0}^{\left[\frac{n-k}{2}\right]}\left(\begin{array}{c}
n-l+r \\
l
\end{array}\right)(n+r-2 l)_{r+k} 2^{n+r-2 l} \\
& \times \int_{-1}^{1} x^{n-k-2 l}\left(1-x^{2}\right)^{k+\lambda-\frac{1}{2}}
\end{aligned}
$$

Now, by virtue of Proposition 3(vii), we have

$$
\int_{-1}^{1} x^{n-k-2 l}\left(1-x^{2}\right)^{k+\lambda-\frac{1}{2}} d x= \begin{cases}0, & n \neq k \quad(\bmod 2) ; \\ \frac{\Gamma\left(k+\lambda+\frac{1}{2}\right) \Gamma\left(\frac{n-k}{2}-l+\frac{1}{2}\right)}{\Gamma\left(\lambda+1-l+\frac{n+k}{2}\right)}, & \text { if } n \equiv k \quad(\bmod 2) .\end{cases}
$$

After certain simplifications, we get

$$
\eta_{n, r}(x)=\frac{\Gamma(\lambda)}{r !} \sum_{\substack{k=0 \\ n \equiv k(\bmod 2)}}^{n} \frac{(\lambda+k)}{\Gamma\left(\lambda+\frac{n+k}{2}+1\right)} \sum_{l=0}^{\left[\frac{n-k}{2}\right]} \frac{(n-l+r) !\left(\frac{n+k}{2}+\lambda\right)_{l}}{\left(\frac{n-k}{2}-l\right) !} \frac{(-1)^{l}}{l !} C_{k}^{(\lambda)}(x) .
$$

Then, by taking $k=n-2 i$ and by virtue of $1(\mathrm{~d})$ and (10), we obtain

$$
\eta_{n, r}(x)=\frac{\Gamma(\lambda)(r+n) !}{r !} \sum_{i=0}^{\left[\frac{n}{2}\right]} \frac{(n+\lambda-2 i)}{i ! \Gamma(n+\lambda-i+1)} C_{n-2 i}^{(\lambda)}(x) \sum_{l=0}^{i} \frac{\langle-i\rangle_{l}\langle-i-\lambda-n\rangle_{l}}{\langle-n-r\rangle_{l}} \frac{(-1)^{l}}{l !}
$$

which on further simplification, we get

$$
\eta_{n, r}(x)=\frac{(r+n) !}{r !(\lambda)_{n+1}} \sum_{i=0}^{\left[\frac{n}{2}\right]} \frac{(n-2 i+\lambda)(\lambda+n)_{i}}{i !}{ }_{2} F_{1}(-i, i-\lambda-n ;-n-r ;-1) C_{n-2 i}^{(\lambda)}(x)
$$

and hence the proof is completed.

\section{Conclusion}

This paper deals with the sums of finite products of Pell polynomials which are represented via hypergeometric functions and explicit computations. It shows how the orthogonal polynomials is utilized very effectively to express the sums and hence the technique of conversion is very much easy and accurate.

\section{Acknowledgements}

The authors take the opportunity to express their heartfelt thanks and gratitude to the anonymous learned reviewers for their valuable comments and suggestions for the improvement and betterment of the manuscript. The review comments and suggestions of the learned reviewer are highly appreciable and praiseworthy.

\section{Authors' contributions}

GKP performed the supervision and validation of the approaches in this research work. AP was responsible for the conceptualization, investigation, methodology, data curation, writing-original draft preparation, writing-reviewing and editing. All authors read and approved the final manuscript.

\section{Funding}

Funding information is not applicable/no funding was received. 


\section{Declarations}

Competing interests

The authors declare that they have no competing interests.

Received: 14 July 2021 Accepted: 13 January 2022

Published online: 31 January 2022

\section{References}

1. Kim, T., Dolgy, D.V., Kim, D.S.: Representing sums of finite products of Chebyshev polynomials of the second kind and Fibonacci polynomials in terms of Chebyshev polynomials. Adv. Stud. Contemp. Math. (Kyungshang) 28(3), 321-335 (2018)

2. Kim, T., Kim, D.S., Won, J., Dolgy, D.V.: Expressing sums of finite products of Chebyshev polynomials of the second kind and of Fibonacci polynomials by several orthogonal polynomials. Mathematics $\mathbf{6}(10), 14$ (2018)

3. Kim, D.S., Kim, T.: On sums of finite products of balancing polynomials. J. Comput. Appl. Math. 377, 112913 (2020)

4. Halici, S.: On the Pell polynomials. Appl. Math. Sci. 5(37), 1833-1838 (2011)

5. Horadam, A.F.: Pell and Pell-Lucas polynomials. Fibonacci Q. 23(1), 7-20 (1985)

6. Koshy, T.: Pell and Pell-Lucas Numbers with Applications. Springer, Berlin(2014)

7. Zhang, Y., Chen, Z.: A new identity involving the Chebyshev polynomials. Mathematics 6(11), 244 (2018)

8. Kim, T., Kim, D.S., Jang, L.C., Jang, G.W.: Sums of finite products of Genocchi functions. Adv. Differ. Equ. 268 , 17 (2017)

9. C. Faber, C., Pandharipande, R.: Hodge integrals and Gromov-Witten theory. Invent. Math. 139, 173-199 (2000)

10. Dunne, G.V., Schubert, C.: Bernoulli number identities from quantum field theory and topological string theory. Commun. Number Theory Phys. 7, 225-249 (2013)

11. Gessel, I.M.: On Miki's identity for Bernoulli numbers. J. Number Theory. 110, 75-82 (2005)

12. Miki, H.: A relation between Bernoulli numbers. J. Number Theory. 10, 297-302 (1978)

13. Panda, G.K., Patra, A.: Exact divisibility by powers of the Pell and Associated Pell numbers. Proc. Math. Sci. (2021). https://doi.org/10.1007/s12044-021-00615-w

14. Shiratani, K., Yokoyama, S.: An application of p-adic convolutions. Mem. Fac. Sic. Kyshu Univ. Ser. A36, 73-83 (1982)

15. Kim, T., Kim, D.S., Dolgy, D.V., Kwon, J.: Sums of finite products of Chebyshev polynomials of two different types. AlMS Math. 6(11), 12528-12542 (2021)

16. Kim, T., Kim, D.S., Dolgy, D.V., Kwon, J.: A note on sums of finite products of Lucas-balancing polynomials. Proc. Jangjeon Math. Soc. 23(1), 1-22 (2020)

17. Kim, D.S., Kim, T., Kim, H.Y., Kwon, J.: Identities of symmetry for Euler polynomials and alternating power sums. Proc. Jangjeon Math. Soc. 24(2), 153-170 (2021)

18. Kim, T., Ryoo, C.S., Kim, D.S., Kwon, J.: A difference of sums of finite products of Lucas-balancing polynomials. Adv. Stud. Contemp. Math. (Kyungshang) 30(1), 121-134 (2020)

19. Kim, T., Kim, D.S., Kim, H.-Y., Lee, H., Jang, L.-C.: A family of associated sequences and their representations by Appell polynomials. Proc. Jangjeon Math. Soc. 23(4), 445-458 (2020)

20. Araci, S.: A new class of Bernoulli polynomials attached to polyexponential functions and related identities. Adv. Stud. Contemp. Math. (Kyungshang) 31(2), 195-2 (2021)

21. https://en.wikipedia.org/wiki/Fallingandrisingfactorials

22. https://en.wikipedia.org/wiki/Hypergeometricfunction

23. https://en.wikipedia.org/wiki/Vandermondeidentity

24. Mason, J.C., Handscomb, D.C.: Chebyshev Polynomials. Chapman and Hall, London (2003)

\section{Publisher's Note}

Springer Nature remains neutral with regard to jurisdictional claims in published maps and institutional affiliations.

\section{Submit your manuscript to a SpringerOpen ${ }^{\circ}$ journal and benefit from:}

- Convenient online submission

Rigorous peer review

- Open access: articles freely available online

High visibility within the field

Retaining the copyright to your article

Submit your next manuscript at $\gg$ springeropen.com 